\title{
A Characterization of Pettis Sets in Dual Banach Spaces
}

\author{
By \\ Minoru MatsudA*
}

\begin{abstract}
In this paper, we give a characterization of Pettis sets in dual Banach spaces in terms of $\delta$ Rademacher trees. This is a generalization of our Theorem 1 in [1].
\end{abstract}

\section{\$1. Introduction}

Throughout this paper $X$ is a real Banach space with topological dual $X^{*}$. The closed unit ball of $X$ is denoted by $B_{X}$. In the sequel, $(\Omega, \Sigma, \mu)$ always denotes a complete probability measure space and $([0,1], \Lambda, \lambda)$ is the Lebesgue measure space on $[0,1]$. For each $(\Omega, \Sigma, \mu)$, a function $f: \Omega \rightarrow X\left(\right.$ resp. $\left.X^{*}\right)$ is said to be scalarly measurable (resp. weak*-measurable) if the real-valued function $\left(x^{*}, f(\omega)\right)$ (resp. $\left.(x, f(\omega))\right)$ is $\mu$-measurable for each $x^{*} \in X^{*}$ (resp. $x \in X)$. We say that a scalarly measurable function $f: \Omega \rightarrow X$ is Pettis integrable if $\left(x^{*}, f(\omega)\right) \in L_{1}(\Omega, \Sigma, \mu)$ for every $x^{*} \in X^{*}$ and moreover for each $E \in \Sigma$ there exists an element $x_{E}$ of $X$ that satisfies

$$
\left(x^{*}, x_{E}\right)=\int_{E}\left(x^{*}, f(\omega)\right) d \mu(\omega)
$$

for every $x^{*} \in X^{*}$. If $f: \Omega \rightarrow X^{*}$ is a weak*-measurable function with bounded range, then we obtain a bounded linear operator $T_{f}: X \rightarrow L_{1}(\Omega, \Sigma, \mu)$ given by $T_{f}(x)=x \circ f$ for every $x \in X$, where $(x \circ f)(\omega)=(x, f(\omega))$ for every $\omega \in \Omega$. The dual operator of $T_{f}$ is denoted by $T_{f}^{4}$.

Let $K$ be a compact Hausdorff space. Then a real-valued function $h$ defined on $K$ is universally measurable if $h$ is $\sigma$-measurable for every Radon probability measure $\sigma$ on $K$. If $f$ is a function from $K$ to $X$, then $f$ is called universally scalarly measurable if the real-valued function $\left(x^{*}, f(k)\right)$ defined on $K$ is universally measurable for each $x^{*} \in X^{*}$.

A subset $C$ of $X$ is said to be a weak Radon-Nikodym set if for any $(\Omega, \Sigma, \mu)$

Communicated by S. Matsuura, January 31, 1991.

1991 Mathematics Subject Classification: 46B22, 46G10.

* Department of Mathematics. Faculty of Science, Shizuoka University, Ohya, Shizuoka 422, Japan. 
and any measure $\alpha: \Sigma \rightarrow X$ for which $\alpha(E) \in \mu(E) \cdot C$ for every $E \in \Sigma$, there exists a Pettis integrable function $g: \Omega \rightarrow C$ such that

$$
\left(x^{*}, \alpha(E)\right)=\int_{E}\left(x^{*}, g(\omega)\right) d \mu(\omega)
$$

for each $E \in \Sigma$ and $x^{*} \in X^{*}$. Let $H$ be a weak*-compact subset of $X^{*}$. Then we always understand in the following that $H$ is topologized by the weak*-topology $\sigma\left(X^{*}, X\right)$. As a general notion of weak*-compact convex weak Radon-Nikodym sets, the following is defined.

Definition. A weak*-compact subset $H$ of $X^{*}$ is called a Pettis set if the identity map $i: H \rightarrow X^{*}$ is universally scalarly measurable.

Then it has been shown in [3] (or [7]) that $H$ is a Pettis set if and only if $w^{*}$ $\operatorname{conv}(H)$ (the weak*-closed convex hull of $H$ ) is a weak Radon-Nikodym set.

Now a sequence $\left\{x_{n}\right\}_{n \geqq 1}$ in $X$ is called a tree if $x_{n}=\left(x_{2 n}+x_{2 n+1}\right) / 2$ for all $n \geqq 1$. Following Riddle and Uh1 [4], we say that a tree $\left\{x_{n}\right\}_{n \geqq 1}$ is a $\delta$-Rademacher tree if there exists a $\delta>0$ such that

$$
\left\|x_{1}\right\| \geqq \delta,\left\|x_{2}-x_{3}\right\| \geqq 2 \delta,\left\|x_{4}-x_{5}+x_{6}-x_{7}\right\| \geqq 4 \delta,
$$

and, in general,

$$
\left\|\sum_{n=2^{m}}^{2^{m+1}-1}(-1)^{n} x_{n}\right\|\left(=\left\|\sum_{i=0}^{2^{m}-1}(-1)^{i} x_{2^{m}+i}\right\|\right) \geqq 2^{m} \delta
$$

for all $m \geqq 0$.

In a series of papers [4], [2] and [1], some attempts to characterize weak*compact convex weak Radon-Nikodym sets of $X^{*}$ in terms of $\delta$-Rademacher trees have been made in various cases (or steps) and the following result (Theorem 1 in [1]) has been obtained.

Theorem A. Let $C$ be a weak*-compact convex subset of $X^{*}$. Then the set $C$ is a weak Radon-Nikodym set if and only if it contains no $\delta$-Rademacher tree.

In this paper we present the following Theorem 1 concerning the characterization of weak*-compact (not necessarily convex) Pettis sets in terms of $\delta$ Rademacher trees, which is an extension of Theorem A. This is the aim of our paper. In the statement (b) of Theorem $1,[0,1]$ is endowed with $\Lambda$ and $\lambda$.

Theorem 1 . Let $H$ be a weak*-compact subset of $X^{*}$. Then the following statements about $H$ are equivalent.

(a) The set $H$ is a Pettis set.

(b) For any weak*-measurable function $f:[0,1] \rightarrow H,\left\{T_{f}^{*}\left(\chi_{A} / \lambda(A)\right)\right.$ : $\lambda(A)>0, A \in \Lambda\}$ contains no $\delta$-Rademacher tree. 
(c) For any $(\Omega, \Sigma, \mu)$ and any weak*-measurable function $f: \Omega \rightarrow H,\left\{T_{f}^{*}\left(\chi_{E} /\right.\right.$ $\mu(E)): \mu(E)>0, E \in \Sigma\}$ contains no $\delta$-Rademacher tree.

\section{§2. Preliminaries}

Before proving Theorem 1, let us recall and prepare some notations and facts that are needed in the process of our proof of Theorem 1.

Let $K$ be a compact Hausdorff space. A sequence of pairs $\left(A_{n}, B_{n}\right)_{n \geqq 1}$ of sets of $K$ with $A_{n} \cap B_{n}=\phi$ for all $n$ is said to be independent if for all $\left\{\varepsilon_{j}\right\}_{1 \leqq j \leqq k}$ with $\varepsilon_{j}=1$ or $-1, \bigcap_{j=1}^{k} \varepsilon_{j} A_{j} \neq \phi$ where $\varepsilon_{j} A_{j}=A_{j}$ if $\varepsilon_{j}=1$ and $\varepsilon_{j} A_{j}=B_{J}$ if $\varepsilon_{j}=-1$. When there exists an independent sequence $\left(A_{n}, B_{n}\right)_{n \geqq 1}$ of closed subsets of $K$, put $\Gamma=\bigcap_{n=1}^{\infty}\left(A_{n} \cup B_{n}\right)$. Then $\Gamma$ is a non-empty compact subset of $K$, since $\left(A_{n}, B_{n}\right)_{n \geqq 1}$ is independent. Define $\phi: \Gamma \rightarrow \Delta\left(=\{0,1\}^{N}\right.$, Cantor space) by $\phi(z)=\left\{t_{n}\right\}_{n \geqq 1}$ where $t_{\mathrm{n}}=1$ if $z \in A_{n}$ and $t_{n}=0$ if $z \in B_{n}$. Then, setting $U_{m}=\left\{t=\left\{t_{n}\right\}_{n \geqq 1} \in \Delta\right.$ : $\left.t_{m}=1\right\}, \phi$ is a continuous surjection that satisfies $\Gamma \cap A_{m}=\phi^{-1}\left(U_{m}\right)$ and $\Gamma \cap B_{m}$ $=\phi^{-1}\left(U_{m}^{c}\right)$ for all $m \geqq 1$. Since $\phi$ is a continuous surjection, we have a Radon probability measure $\gamma$ on $\Gamma$ such that $\phi(\gamma)$ (the image measure of $\gamma$ by $\phi)=v$ (the normalized Haar measure on $\Delta)$ and $\left\{f \circ \phi: f \in L_{1}\left(\Delta, \Sigma_{v}, v\right)\right\}=L_{1}\left(\Gamma, \Sigma_{\gamma}, \gamma\right)$ (cf. (1-2-5) in [7]), where $\Sigma_{v}$ (resp. $\Sigma_{\gamma}$ ) is the family of all $v$ (resp. $\gamma$ )-measurable subsets of $\Delta$ (resp. $\Gamma$ ).

Furthermore, consider a function $\rho: \Delta \rightarrow[0,1]$ defined by $\rho(t)=\sum_{n=1}^{\infty} t_{n} / 2^{n}$, where $t=\left\{t_{n}\right\}_{n \geqq 1} \in \Delta$. Then we easily obtain that $\rho$ is a continuous surjection satisfying $\rho(v)=\lambda$ and $\left\{u \circ \rho: u \in L_{1}([0,1], \Lambda, \lambda)\right\}=L_{1}\left(\Delta, \Sigma_{v}, v\right)$.

Under these preparations we first note the following lemma, which has been essentially suggested in the proof of $(b) \Rightarrow$ (a) of Theorem (7-3-7) in [7]. But, for the sake of completeness, we dare state it in a more explicit form suitable for our subsequent argument and give its simple proof.

Lemma. Let $S$ be the linear operator on $L_{1}([0,1], \Lambda, \lambda)$ given by $S(u)=$ $u \circ \rho \circ \phi$ for every $u \in L_{1}([0,1], \Lambda, \lambda)$. Then the following statements hold.

(a) The linear operator $S$ is a surjective isometry from $L_{1}([0,1], \Lambda, \lambda)$ to $L_{1}\left(\Gamma, \Sigma_{\gamma}, \gamma\right)$.

(b) For every $g \in L_{x}\left(\Gamma, \Sigma_{\gamma}, \gamma\right), S^{*}(g)(\rho(\phi(z)))=g(z)$-a.e. on $\Gamma$, where $S^{*}$ is the dual operator of $S$.

(c) For $g_{1}$ and $g_{2}$ in $L_{\infty}\left(\Gamma, \Sigma_{\gamma}, \gamma\right), S^{*}\left(g_{1} \cdot g_{2}\right)=S^{*}\left(g_{1}\right) \cdot S^{*}\left(g_{2}\right)$ in $L_{\infty}([0,1]$, $\Lambda, \lambda)$.

Proof. Let us first show the statement (a). As for the isometry of $S$, it easily follows from the fact that 


$$
\begin{gathered}
\int_{\Gamma}|S(u)(z)| d \gamma(z)=\int_{\Gamma}|u(\rho(\phi(z)))| d \gamma(z) \\
\quad=\int_{\Delta}|u(\rho(t))| d v(t)=\int_{[0,1]}|u(s)| d \lambda(s)
\end{gathered}
$$

using the change-of-variables formula. As for the surjectivity of $S$, suppose the contrary. Then $\left\{S(u): u \in L_{1}([0,1], \Lambda, \lambda)\right\}$ is a proper closed subspace of $L_{1}(\Gamma$, $\left.\Sigma_{\gamma}, \gamma\right)$. Hence, by the Hahn-Banach theorem, there exists a non-zero element $\mathrm{g}$ of $L_{\infty}\left(\Gamma, \Sigma_{\gamma}, \gamma\right)$ such that $\int_{\Gamma} g(z) S(u)(z) d \gamma(z)=0$ for every $u \in L_{1}([0,1], \Lambda, \lambda)$. That is, $\int_{\Gamma} g(z) u(\rho(\phi(z))) d \gamma(z)=0$ for every $u \in L_{1}([0,1], \Lambda, \lambda)$. Since $\{u \circ \rho$ : $\left.u \in L_{1}([0,1], \Lambda, \lambda)\right\}=L_{1}\left(\Delta, \Sigma_{v}, v\right)$ as stated above, $\int_{\Gamma} g(z) f(\phi(z)) d \gamma(z)=0$ for every $f \in L_{1}\left(\Delta, \Sigma_{v}, v\right)$ which means that $g=0$ in $L_{\infty}\left(\Gamma, \Sigma_{\gamma}, \gamma\right)$ since $\{f \circ \phi: f \in$ $\left.L_{1}\left(\Delta, \Sigma_{v}, v\right)\right\}=L_{1}\left(\Gamma, \Sigma_{\gamma}, \gamma\right)$ as noted above. This is a contradiction.

To prove (b), it suffices for us to show that

$$
\int_{\Gamma} S^{*}(g)(\rho(\phi(z))) h(z) d \gamma(z)=\int_{\Gamma} g(z) h(z) d \gamma(z)
$$

for every $h \in L_{1}\left(\Gamma, \Sigma_{\gamma}, \gamma\right)$. For each $h \in L_{1}\left(\Gamma, \Sigma_{\gamma}, \gamma\right)$, in virtue of (a), there exists an element $u$ of $L_{1}([0,1], \Lambda, \lambda)$ such that $h=u \circ \rho \circ \phi$ in $L_{1}\left(\Gamma, \Sigma_{\gamma}, \gamma\right)$. Then we have

$$
\begin{aligned}
& \int_{[0,1]} S^{*}(g)(s) u(s) d \lambda(s)=\int_{\Gamma} g(z) S(u)(z) d \gamma(z) \\
& =\int_{\Gamma} g(z) u(\rho(\phi(z))) d \gamma(z)=\int_{\Gamma} g(z) h(z) d \gamma(z) .
\end{aligned}
$$

On the other hand, we have

$$
\begin{aligned}
& \int_{[0,1]} S^{*}(g)(s) u(s) d \lambda(s)=\int_{[0,1]} S^{*}(g)(s) u(s) d(\rho(\phi(\gamma)))(s) \\
& \quad=\int_{\Gamma} S^{*}(g)(\rho(\phi(z))) u(\rho(\phi(z))) d \gamma(z)=\int_{\Gamma} S^{*}(g)(\rho(\phi(z))) h(z) d \gamma(z) .
\end{aligned}
$$

Hence we have the desired equality for each $h \in L_{1}\left(\Gamma, \Sigma_{\gamma}, \gamma\right)$. This completes the proof of (b). Finally, by virtue of (b), the statement (c) follows easily. So the proof of Lemma is completed.

\section{§3. Proof of Theorem $\mathbb{1}$}

In [3] and [7], the following Theorem $\mathbb{B}$ has been obtained concerning the characterization of Pettis sets in dual Banach spaces. 
Theorem B. Let $H$ be a weak*-compact subset of $X^{*}$. Then the following statements about $H$ are equivalent.

(a) The set $H$ is a Pettis set.

(b) Each sequence of $A$ has a pointwise convergent subsequence on $H$, where $A=\left\{x_{\mid H}: x \in B_{X}\right\}$ (that is, every sequence $\left\{x_{n}\right\}_{n \geqq 1}$ in $B_{X}$ has a subsequence $\left\{x_{n(k)}\right\}_{k \geqq 1}$ such that for every $x^{*} \in H, \lim _{k \rightarrow \infty}\left(x^{*}, x_{n(k)}\right)$ exists $)$.

(c) The set $w^{*}$-conv $(H)$ is a weak Radon-Nikodym set.

We are now in a position to prove Theorem 1, making use of Theorems A, $B$ and Lemma.

Proof of Theorem 1. Let us first show that (a) implies (c). In virtue of Theorems $\mathrm{A}$ and $\mathrm{B}$, the proof of this part is simple. Let $H$ be a Pettis set and suppose that (c) fails. Then there exist a complete probability measure space $(\Omega$, $\Sigma, \mu)$ and a weak*-measurable function $f: \Omega \rightarrow H$ such that $\left\{T_{f}^{\star}\left(\chi_{E} / \mu(E)\right)\right.$ : $\mu(E)>0, E \in \Sigma\}$ contains a $\delta$-Rademacher tree. For each $E \in \Sigma$, we have that $\left(T_{f}\left(\chi_{E}\right), x\right)=\left(\chi_{E}, T_{f}(x)\right)=\int_{E}(x, f(\omega)) d \mu(\omega)$ for every $x \in X$. Hence, by the separation theorem, it holds that $T_{f}^{+}\left(\chi_{E}\right) \in \mu(E) \cdot w^{*}$-conv $(H)$ for each $E \in \Sigma$, since $f(\Omega) \subset H$. So $w^{*}$-conv $(H)$ contains a $\delta$-Rademacher tree by the assumption. Thus, by Theorem $\mathrm{B}, w^{*}$-conv $(H)$ is a weak Radon-Nikodym set containing a $\delta$-Rademacher tree, which is contradictory to Theorem A.

Next let us prove that (b) implies (a). This is the crucial part of our proof. Suppose that $H$ is not a Pettis set. Then we are going to construct a weak ${ }^{*}$ measurable function $h:[0,1] \rightarrow H$ such that $\left\{T_{h}^{4}\left(\chi_{A} / \lambda(A)\right): \lambda(A)>0, A \in \Lambda\right\}$ contains a $\delta$-Rademacher tree for an appropriate positive number $\delta$. To this end, invoke Theorem $\mathrm{B}$ to conclude that there exists a sequence $\left\{x_{n}\right\}_{n \geqq 1}$ in $B_{X}$ having no pointwise convergent subsequence on $H$. Then, by the celebrated argument of Rosenthal (cf. [5] or [6]), we have a subsequence $\left\{x_{n(k)}\right\}_{k \geqq 1}$ of $\left\{x_{n}\right\}_{n \geq 1}$ and real numbers $r$ and $\eta$ with $\eta>0$ such that putting $A_{k}=\left\{x^{*} \in H:\left(x^{*}\right.\right.$, $\left.\left.x_{n(k)}\right) \leqq r\right\}$ and $B_{k}=\left\{x^{*} \in H:\left(x^{*}, x_{n(k)}\right) \geqq r+2 \eta\right\}$, then $\left(A_{k}, B_{k}\right)_{k \geqq 1}$ is an independent sequence of pairs of closed subsets of $H$. Put $\Gamma=\bigcap_{k=1}^{\infty}\left(A_{k} \cup B_{k}\right) \subset H$. Then, by Lemma above, we have a complete probability measure space $(\Gamma$, $\left.\Sigma_{\gamma}, \gamma\right)$ and a surjective isometry $S: L_{1}([0,1], \Lambda, \lambda) \rightarrow L_{1}\left(\Gamma, \Sigma_{\gamma}, \gamma\right)$ such that $S^{*}(g)\left(\rho\left(\phi\left(x^{*}\right)\right)\right)=g\left(x^{*}\right) \gamma$-a.e. on $\Gamma$ for every $g \in L_{x}\left(\Gamma, \Sigma_{\gamma}, \gamma\right)$ (Here, functions $\rho$ and $\phi$ are the same ones as stated in $\$ 2$ ).

Now, for each $x \in B_{X}$, consider a function $f_{x}$ on $\Gamma$ given by $f_{x}\left(x^{*}\right)=\left(x^{*}, x\right)$ for every $x^{*} \in \Gamma$. Then $\left\{f_{x}: x \in B_{X}\right\} \subset C(\Gamma)$ (the Banach space of all real-valued continuous functions on $\Gamma)$. Let $p$ be a lifting of $L_{x}([0,1], \Lambda, \lambda)$. For each fixed $s \in[0,1]$, we associate a following bounded linear functional $L_{s}$ on $C(\Gamma)$ : $L_{s}(f)=p\left(S^{*}(f)\right)(s)$ for every $f \in C(\Gamma)$. Then, in virtue of (c) in Lemma, $L_{\mathrm{s}}$ is multiplicative and so there exists a unique point $x^{*} \in \Gamma$ such that $L_{s}(f)=f\left(x^{*}\right)$ for every $f \in C(\Gamma)$. Then define $h:[0,1] \rightarrow \Gamma$ by $h(s)=x^{*}$ for each $s \in[0,1]$. This 
means that $f(h(s))=p\left(S^{*}(f)\right)(s)$ for every $f \in C(\Gamma)$. Hence, in particular, $f_{x}(h(s))=p\left(S^{*}\left(f_{x}\right)\right)(s)$ for every $x \in B_{X}$. That is, we have that $(x, h(s))=$ $p\left(S^{*}\left(f_{x}\right)\right)(\mathrm{s})$ for every $s \in[0,1]$ and $x \in B_{X}$. So the function $h$ is weak*-measurable. Moreover, we have that for each $A \in \Lambda$,

$$
\begin{aligned}
& \int_{A}(x, h(s)) d \lambda(s)=\int_{A} P\left(S^{*}\left(f_{x}\right)\right)(s) d \lambda(s)=\int_{A} S^{*}\left(f_{x}\right)(s) d \lambda(s) \\
& =\int_{A} S^{*}\left(f_{x}\right)(s) d(\rho(\phi(\gamma)))(s)=\int_{\phi^{-1}\left(\rho^{-1}(A)\right)} S^{*}\left(f_{x}\right)\left(\rho\left(\phi\left(x^{*}\right)\right)\right) d \gamma\left(x^{*}\right) \\
& =\int_{\phi^{-1}\left(\rho^{-1}(A)\right)} f_{x}\left(x^{*}\right) d \gamma\left(x^{*}\right) \quad \text { (by (b) of Lemma) } \\
& =\int_{\phi^{-1}\left(\rho^{-1}(A)\right)}\left(x^{*}, x\right) d \gamma\left(x^{*}\right) \ldots(*) .
\end{aligned}
$$

Consider a measure $\alpha: \Lambda \rightarrow X^{*}$ defined for each $A \in \Lambda$ by putting

$$
(\alpha(A), x)=\int_{A}(x, h(s)) d \lambda(s)
$$

for every $x \in X$. Then it holds that $\alpha(A)=T_{h}^{*}\left(\chi_{A}\right)$ for every $A \in \Lambda$, since $(\alpha(A), x)=\int_{A}(x, h(s)) d \lambda(s)=\left(\chi_{A}, T_{h}(x)\right)=\left(T_{h}^{*}\left(\chi_{A}\right), x\right)$ for every $x \in X$. Put $x_{2^{m}+i}^{+}=2^{m} \alpha\left(I_{m, i}\right)\left(m \geqq 0,0 \leqq i \leqq 2^{m}-1\right)$, where $I_{m, i}=\left[i / 2^{m},(i+1) / 2^{m}\right]$. Then we easily know that $x_{n}^{*}=\left(x_{2 n}^{*}+x_{2 n+1}^{*}\right) / 2$ for all $n \geqq 1$ and the sequence $\left\{x_{n}^{*}\right\}_{n \geqq 1}$ is contained in $\left\{T_{h}^{*}\left(\chi_{A} / \lambda(A)\right): \lambda(A)>0, A \in \Lambda\right\}$. Hence, to complete the proof of this part, it suffices for us to show that the sequence $\left\{x_{n}^{*}\right\}_{n \geqq 1}$ contains a $\delta$-Rademacher tree for an appropriate positive number $\delta$.

To see this, let us first relabel $\left\{I_{m, i}\right\}\left(m \geqq 0,0 \leqq i \leqq 2^{m}-1\right)$ by $I_{0,0}=I(=$ $[0,1]), I_{1,0}=I(0), I_{1,1}=I(1), I_{2,0}=I(0,0), I_{2,1}=I(0,1), I_{2,3}=I(1,0), I_{2,4}=$ $I(1,1)$, etc. That is, $I_{0,0}=I$ and if $I_{m, i}=I\left(a_{1}^{(i)}, \ldots, a_{m}^{(i)}\right)\left(m \geqq 1,0 \leqq i \leqq 2^{m}-1\right)$, then

$$
I_{m+1,2 i}=I\left(a_{1}^{(i)}, \ldots, a_{m}^{(i)}, 0\right)
$$

and

$$
I_{m+1,2 i+1}=I\left(a_{1}^{(i)}, \ldots, a_{m}^{(i)}, 1\right) .
$$

Here $\left\{a_{j}^{(i)}\right\}_{1 \leqq j \leqq m}$ denotes a sequence consisting of 0 or 1 and $\left\{\left(a_{1}^{(i)}, \ldots, a_{m}^{(i)}\right): 0 \leqq\right.$ $\left.i \leqq 2^{m}-1\right\}=\left\{\left(a_{1}, \ldots, a_{m}\right): a_{j}=0\right.$ or $\left.a_{j}=1\right\}$. Then we have that for every $\left\{a_{j}\right\}_{1 \leqq j \leqq m}$ with $a_{j}=0$ or 1

$$
U_{1}^{\varepsilon(1)} \cap \ldots \cap U_{m}^{\varepsilon(m)}=\rho^{-1}\left(I\left(a_{1}, \ldots, a_{m}\right)\right),
$$

where $\varepsilon(j)=1$ if $a_{j}=1$ and $\varepsilon(j)=\mathrm{c}$ (complement) if $a_{j}=0$. Hence we have that for every $\left\{a_{j}\right\}_{1 \leqq j \leqq m}$ with $a_{j}=0$ or 1 , 


$$
\begin{aligned}
& \left(\alpha\left(I\left(a_{1}, \ldots, a_{m}\right)\right), x\right)=\int_{I\left(a_{1}, \ldots, a_{m}\right)}(x, h(s)) d \lambda(s) \\
& \quad=\int_{\phi^{-1}\left(\rho^{-1}\left(I\left(a_{1}, \ldots, a_{m}\right)\right)\right)}\left(x^{*}, x\right) d \gamma\left(x^{*}\right) \quad \text { (by }\left(^{*}\right) \text { above) } \\
& =\int_{\phi^{-1}\left(U_{1}^{\varepsilon(1)} \ldots \cap U_{m}^{\varepsilon(m)}\right)}\left(x^{*}, x\right) d \gamma\left(x^{*}\right)
\end{aligned}
$$

for all $x \in X$. Then this easily yields that

$$
\begin{aligned}
& \sum_{i=0}^{2^{m}-1}\left(\alpha\left(I\left(a_{1}^{(i)}, \ldots, a_{m}^{(i)}, 0\right)\right), x_{n(m+1)}\right) \\
& \quad=\int_{\phi^{-1}\left(U_{m+1}^{i}\right)}\left(x^{*}, x_{n(m+1)}\right) d \gamma\left(x^{*}\right)=\int_{\Gamma \cap B_{m+1}}\left(x^{*}, x_{n(m+1)}\right) d \gamma\left(x^{*}\right)
\end{aligned}
$$

and

$$
\begin{aligned}
& \sum_{i=0}^{2^{m}-1}\left(\alpha\left(I\left(a_{1}^{(i)}, \ldots, a_{m}^{(i)}, 1\right)\right), x_{n(m+1)}\right) \\
& \quad=\int_{\phi^{-1}\left(U_{m+1}\right)}\left(x^{*}, x_{n(m+1)}\right) d \gamma\left(x^{*}\right)=\int_{\Gamma \cap A_{m+1}}\left(x^{*}, x_{n(m+1)}\right) d \gamma\left(x^{*}\right) .
\end{aligned}
$$

Thus we have that for every $m \geqq 0$,

$$
\begin{aligned}
& \left\|\sum_{i=0}^{2^{m+1}-1}(-1)^{i} x^{*} 2^{m+1}+i\right\| \\
& \geqq\left(\sum_{i=0}^{2^{m+1}-1}(-1)^{l} x_{2^{m+1}+i}, x_{n(m+1)}\right)
\end{aligned}
$$

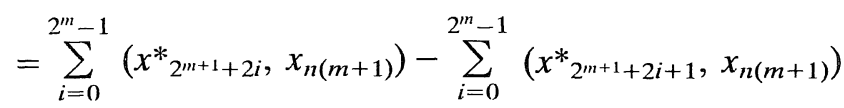

$$
\begin{aligned}
& =2^{m+1} \cdot\left\{\sum_{i=0}^{2^{m}-1}\left(\alpha\left(I\left(a_{1}^{(i)}, \ldots, a_{m}^{(i)}, 0\right)\right), x_{n(m+1)}\right)\right. \\
& \left.-\sum_{i=0}^{2^{m}-1}\left(\alpha\left(I\left(a_{1}^{(i)}, \ldots, a_{m}^{(i)}, 1\right)\right), x_{n(m+1)}\right)\right\} \\
& =2^{m+1} \cdot\left\{\int_{\Gamma \cap B_{m+1}}\left(x^{*}, x_{n(m+1)}\right) d \gamma\left(x^{*}\right)\right. \\
& \left.-\int_{\Gamma \cap A_{m+1}}\left(x^{*}, x_{n(m+1)}\right) d \gamma\left(x^{*}\right)\right\} \\
& \geqq 2^{m+1} \cdot\{(r+2 \eta) / 2-r / 2\}=2^{m+1} \eta \text {. }
\end{aligned}
$$


In the case where $x_{1}^{+}(=\alpha(I)) \neq 0$, setting $\delta=\min \left\{\left\|x_{1}^{*}\right\|, \eta\right\}(>0),\left\{x_{n}^{+}\right\}_{n \geqq 1}$ itself is a $\delta$-Rademacher tree contained in $\left\{T_{h}^{+}\left(\chi_{A} / \lambda(A)\right): \lambda(A)>0, A \in \Lambda\right\}$, which is contradictory to (b).

Next consider the case where $x_{1}^{t}=0$. By the inequality proved above, it holds that $\left\|x_{2}^{\prime}-x_{3}^{*}\right\| \geqq 2 \eta$. Hence one of $x_{2}^{+}$and $x_{3}^{*}$ does not vanish. So, without loss of generality, we may assume that $x_{2}^{4}(=\alpha([0,1 / 2])) \neq 0$. Let $\left\{J_{m, i}\right\}(m \geqq 0$, $\left.0 \leqq i \leqq 2^{m}-1\right)$ be a sequence of closed intervals in $[0,1 / 2]$ given by $J_{m, i}=$ $\left[i / 2^{m+1},(i+1) / 2^{m+1}\right]$. Then, putting $y_{2^{m}+i}^{+}=2^{m+1} \alpha\left(J_{m, i}\right)\left(m \geqq 0,0 \leqq i \leqq 2^{m}-1\right)$, $\left\{y_{n}^{+}\right\}_{n \geqq 1}$ is a tree contained in $\left\{T_{h}^{+}\left(\chi_{A} / \lambda(A)\right): \lambda(A)>0, A \in \Lambda\right\}$. Relabel $\left\{J_{m, i}\right\}$ $\left(m \geqq 0,0 \leqq i \leqq 2^{m}-1\right)$ by $J_{0,0}=J(=[0,1 / 2]), J_{1,0}=J(0), J_{1,1}=J(1), J_{2,0}=$ $J(0,0), J_{2,1}=J(0,1), J_{2,3}=J(1,0), J_{2,4}=J(1,1)$, etc. Then it holds that for every $\left\{a_{j}\right\}_{1 \leqq j \leqq m}$ with $a_{j}=0$ or 1 ,

$$
U_{1}^{c} \cap U_{2}^{\varepsilon(1)} \cap \ldots \cap U_{m+1}^{\varepsilon(m)}=\rho^{-1}\left(J\left(a_{1}, \ldots, a_{m}\right)\right)
$$

in the same notation as above. Hence, by the same argument as above we have

$$
\begin{aligned}
& \sum_{i=0}^{2^{m}-1}\left(\alpha\left(J\left(a_{1}^{(i)}, \ldots, a_{m}^{(l)}, 0\right)\right), x_{n(m+2)}\right) \\
& =\int_{\phi^{-1}\left(U_{1} \cap U_{m+2}\right)}\left(x^{*}, x_{n(m+2)}\right) d \gamma\left(x^{*}\right) \\
& =\int_{\Gamma \cap B_{1} \cap B_{m+2}}\left(x^{*}, x_{n(m+2)}\right) d \gamma\left(x^{*}\right)
\end{aligned}
$$

and

$$
\begin{aligned}
& \sum_{i=0}^{2^{m}-1}\left(\alpha\left(J\left(a_{1}^{(i)}, \ldots, a_{m}^{(i)}, 1\right)\right), x_{n(m+2)}\right) \\
& =\int_{\phi^{-1}\left(U_{1}^{c} \cap U_{m+2}\right)}\left(x^{*}, x_{n(m+2)}\right) d \gamma\left(x^{*}\right) \\
& =\int_{\Gamma \cap B_{1} \cap A_{m+2}}\left(x^{*}, x_{n(m+2)}\right) d \gamma\left(x^{*}\right) .
\end{aligned}
$$

Consequently, we have that for every $m \geqq 0$,

$$
\begin{aligned}
& \left\|\sum_{i=0}^{2^{m+1}-1}(-1)^{l} y^{*} 2^{m+1}+i\right\| \\
& \geqq\left(\sum_{i=0}^{2^{m+1}-1}(-1)^{i} y^{*} 2^{m+1}+i, x_{n(m+2)}\right) \\
& =2^{m+2} \cdot\left\{\int_{\Gamma \cap B_{1} \cap B_{m+2}}\left(x^{*}, x_{n(m+2)}\right) d \gamma\left(x^{*}\right)\right.
\end{aligned}
$$




$$
\begin{gathered}
\left.-\int_{\Gamma \cap B_{1} \cap A_{m+2}}\left(x^{*}, x_{n(m+2)}\right) d \gamma\left(x^{*}\right)\right\} \\
\geqq 2^{m+2} \cdot\{(r+2 \eta) / 4-r / 4\}=2^{m+1} \eta .
\end{gathered}
$$

So, setting $\delta=\min \left\{\left\|x_{2}^{*}\right\|, \eta\right\} \quad(>0)$, we obtain a $\delta$-Rademacher tree $\left\{y_{n}^{*}\right\}_{n \geqq 1}$ contained in $\left\{T_{h}^{+}\left(\chi_{A} / \lambda(A)\right): \lambda(A)>0, A \in \Lambda\right\}$, which is contradictory to (b). Hence we complete the proof of the fact that (b) implies (a). Moreover, the fact that (c) implies (b) is obvious and so the proof of Theorem 1 is completed.

Remark 1. Consider the case where $\mathrm{H}$ is a weak*-compact convex Pettis set. Then, by Theorem $\mathrm{B}$, the set $H$ is a weak Radon-Nikodym set. Hence, in virtue of Theorem 1 , the set $H$ is a weak Radon-Nikodym set if and only if for any weak*-measurable function $f:[0,1] \rightarrow H,\left\{T_{f}^{+}\left(\chi_{A} / \lambda(A)\right): \lambda(A)>0, A \in \Lambda\right\}$ contains no $\delta$-Rademacher tree (Of course, this result follows more easily even from our proof of Theorem 1 in [1]). In particular, the set $H$ is a weak RadonNikodym set if and only if it contains no $\delta$-Rademacher tree (Theorem A), since $T_{f}^{+}\left(\chi_{A}\right) \in \lambda(A) \cdot H$ in this case.

Remark 2. Without invoking Lemma, the equivalence between the statements (a) and (c) of Theorem 1 can be proved. In fact, we have only to show that (c) implies (a) whose proof goes as follows. Suppose that (a) fails. Then, by the first part of the proof of the fact that (b) implies (a) in Theorem 1, we have a complete probability measure space $\left(\Gamma, \Sigma_{\gamma}, \gamma\right)$ and a continuous surjection $\phi$ : $\Gamma \rightarrow \Delta$ such that $\phi(\gamma)=v$. Let $h: \Gamma \rightarrow H$ given by $h\left(x^{*}\right)=x^{*}$ for every $x^{*} \in \Gamma$. Then the function $h$ is weak*-measurable and $\left(T_{h}^{*}\left(\chi_{E}\right), x\right)=\int_{E}\left(x^{*}, x\right) d \gamma\left(x^{*}\right)$ for every $E \in \Sigma_{\gamma}$ and $x \in X$. Define a sequence $\left\{x_{n}^{+}\right\}_{n \geqq 1}$ by $x_{1}^{*}=T_{h}^{+}\left(\chi_{\Gamma}\right)$ and if $x_{2^{m}+i}^{\star}$ $=2^{m} \cdot T_{h}^{\mathrm{k}}\left(\chi_{\Gamma \cap \varepsilon_{1}^{(i)} A_{1} \cap \ldots \cap \varepsilon_{m}^{(t)} \mathrm{A}_{\mathrm{m}}}\right)\left(m \geqq 1,0 \leqq i \leqq 2^{m}-1\right)$, then

$$
x_{2^{m+1}+2 i}^{+}=2^{m+1} \cdot T_{h}^{+}\left(\chi_{\Gamma \cap \varepsilon_{1}^{(l)} A_{1} \cap \ldots \cap \varepsilon_{m}^{(l)} A_{m} \cap B_{m+1}}\right)
$$

and

$$
x_{2^{m+1}+2 i+1}^{+}=2^{m+1} \cdot T_{h}^{+}\left(\chi_{\Gamma \cap \varepsilon_{1}^{(l)} A_{1} \cap \ldots \cap \varepsilon_{m}^{(l)} A_{m} \cap A_{m+1}}\right) .
$$

Here $\left\{\varepsilon_{j}^{(i)}\right\}_{1 \leqq j \leqq m}$ denotes a sequence of 1 or -1 and $\left\{\left(\varepsilon_{1}^{(i)}, \ldots, \varepsilon_{m}^{(i)}\right): 0 \leqq i \leqq 2^{m}-\right.$ $1\}=\left\{\left(\varepsilon_{1}, \ldots, \varepsilon_{m}\right): \varepsilon_{j}=1\right.$ or -1$\}$ for all $m \geqq 1$. Then, by the same argument as above, the sequence $\left\{x_{n}^{+}\right\}_{n \geqq 1}$ contains a $\delta$-Rademacher tree for an appropriate positive number $\delta$ and so $\left\{T_{h}^{*}\left(\chi_{E} / \gamma(E)\right): \gamma(E)>0, E \in \Sigma_{\gamma}\right\}$ contains a $\delta$ Rademacher tree, which is contradictory to (c). Hence the proof is completed.

Finally, let us note that Theorem 1 and the results of [3] or [7] give the following theorem. 
Theorem 2. Let $H$ be a weak*-compact subset of $X^{*}$. Then the following statements about $H$ are equivalent.

(a) The set $H$ is a Pettis set.

(b) Every sequence in $B_{X}$ has a pointwise convergent subsequence on $H$.

(c) For every $x^{* *} \in X^{* *}$ and every weak*-compact subset $M$ of $H$, the function $x^{* *}$ restricted to $M$ has a point of continuity.

(d) For any weak ${ }^{*}$-measurable function $f:[0,1] \rightarrow H,\left\{T_{f}^{4}\left(\chi_{A}\right): A \in \Lambda\right\}$ is relatively norm compact.

(e) The set $w^{*}$-conv $(H)$ is a weak Radon-Nikodym set..

(f) For any weak*-measurable function $f:[0,1] \rightarrow H, \quad\left\{T_{f}^{+}\left(\chi_{\mathrm{A}} / \lambda(A)\right)\right.$ : $\lambda(A)>0, A \in \mathbb{A}\}$ contains no $\delta$-Rademacher tree.

\section{References}

[1] Matsuda, M., A characterization of weak Radon-Nikodym sets in dual Banach spaces, Publ. RIMS, Kyoto Univ., 22 (1986), 551-559.

[2] Riddle. L. H., The geometry of weak Radon-Nikodym sets in dual Banach spaces, Proc. Amer. Math. Soc., 86 (1982), 433-438.

[3] Riddle, L. H. and Saab, E.. On functions that are universally Pettis integrable. Illinois J. Math., 29 (1985), 509-531.

[4] Riddle, L.H. and Uhl, Jr, J.J., Martingales and the fine line between Asplund spaces and spaces not containing a copy of $l_{1}$, Lecture Notes in Math. Springer, 939 (1982), 145-156.

[5] Rosenthal, H.P., A characterization of Banach spaces containing $l_{1}$, Proc. Nat. Acad. Sci., 71 (1974), 2411-2413.

[6] Some recent discoveries in the isomorphic theory of Banach spaces, Bull. Amer. Math. Soc., 84 (1978), 803-831.

[7] Talagrand, M.. Pettis integral and measure theory, Mem. Amer. Math. Soc., 307 (1984). 